\title{
Fast Frequency Sweep for Building-Block Connections in Microwave Filter Analysis via the Reduced-Basis Method
}

\author{
Alba Galán and Valentín de la Rubia
}

\begin{abstract}
Current design of microwave filters relies on intensive computer simulations to accurately predict the electromagnetic behavior of microwave circuits. Typically, global optimization techniques are taken into account and reduced knowledge of the actual electromagnetics is considered within computer-aided design. To this end, microwave filter development results in a rather time-consuming engineering task.

In this work, we address a methodology for fast frequency analysis in microwave circuits in order to speed up full-wave simulations. Appropriate Finite Element solution to time-harmonic Maxwell's equations in the frequency band of interest is involved and a reliable reduced-order model is obtained via the ReducedBasis Method. This time, the analysis domain is decomposed into building-blocks and a reduced-order model for fast frequency sweep is carried out for each block. The electromagnetics within each block is described in terms of a Generalised Impedance Matrix (GIM) transfer function. In order to get the frequency response of the whole microwave circuit, the GIMs for each block should be connected appropriately. This process has been traditionally done frequency by frequency and can be considered a bottleneck in Domain Decomposition (DD) approaches. In this work, we take into account a further fast frequency sweep for the building-block connection problem. As a result, a speed-up in the simulation time in microwave circuits is achieved as the bottleneck in the DD approach is completely removed.

Index Terms-Computer aided engineering, finite element methods, Galerkin method, reduced basis methods, reduced order systems.
\end{abstract}

\section{INTRODUCTION}

Finite Element Methods (FEM) have proven to be helpful in solving the time-harmonic Maxwell's equations for the analysis of microwave devices. Domain Decomposition (DD) approaches have emerged as a possible solution to speedup computer-aided design of microwave circuits, since computational resources can be directly applied to those blocks in the analysis domain that evolve in the optimization loop [1], [2]. The frequency response for each block can definitely be carried out in an efficient way by means of model-order reduction [3]. However, in this DD setting, still the connection problem needs to be solved frequency by frequency in order to resemble the response of the whole microwave device, which we refer to as direct connection problem, resulting in a bottleneck for this approach. We address this issue and propose a solution within the Reduced-Basis Method [4], [5]

This work is motivated by an Arcioni and Conciauro paper in late 90s [6]. In that paper, Arcioni and Conciauro proposed a straightforward technique, especially suitable for BI-RME method [7], to solve for the connection problem. We follow their guidance and extend its applicability within the ReducedBasis Method.

\section{Problem Statement}

Let us consider a two-port microwave circuit, namely, a microwave filter, where Maxwell's equations describe the physical behavior. We are interested in the filter transfer function representing the electromagnetic phenomena in this circuit, namely, a Generalized Impedance Matrix (GIM) transfer function. After discretization of the time-harmonic Maxwell's equations by means of FEM, we get the following linear dynamical system representation of the microwave circuit:

$$
\left(\begin{array}{c}
v_{1} \\
v_{2} \\
0
\end{array}\right)=\left(\begin{array}{ccc}
0 & 0 & j b_{1}^{T} \\
0 & 0 & j b_{2}^{T} \\
s j b_{1} & s j b_{2} & S+s^{2} T
\end{array}\right)\left(\begin{array}{l}
i_{1} \\
i_{2} \\
x
\end{array}\right),
$$

where $S$ and $T$ are the stiffness and mass matrices, $x$ stands for the state-space variable and $s$ is the complex frequency. Once the state-space variable, which represents the FEM discretization of the electric field, is eliminated from equation (1), the GIM transfer function arises, thus,

$$
\left(\begin{array}{l}
v_{1} \\
v_{2}
\end{array}\right)=-s\left(\begin{array}{l}
j b_{1}^{T} \\
j b_{2}^{T}
\end{array}\right)\left(S+s^{2} T\right)^{-1}\left(\begin{array}{ll}
j b_{1} & j b_{2}
\end{array}\right)\left(\begin{array}{l}
i_{1} \\
i_{2}
\end{array}\right) .
$$

It should be noted that the dynamical system (1) has a rather large state-space, thus solving this system frequency by frequency cannot be afforded. In this regard, we propose a reduced-order model to carry out the frequency sweep in an efficient way and, ultimately, to get a circuit theory representation within Electromagnetics.

\section{A. Model Order Reduction}

In order to propose an effective reduced-order model to obtain physical insight from Electromagnetics in the frequency band of interest, we had better make explicit the different state-space variables that come up in place in order to get the impedance matrix transfer function of the two-port microwave circuit, namely, $x_{1}$ and $x_{2}$, each of these arising whenever we are solving the two unitary problems needed to characterize the electromagnetic behavior in the microwave device for any 
excitation. As a result, the following linear dynamical system arises:

$$
\left(\begin{array}{c}
v_{1} \\
v_{2} \\
0 \\
0
\end{array}\right)=\left(\begin{array}{cccc}
0 & 0 & j b_{1}^{T} & j b_{1}^{T} \\
0 & 0 & j b_{2}^{T} & j b_{2}^{T} \\
s j b_{1} & s j b_{1} & S+s^{2} T & 0 \\
s j b_{2} & s j b_{2} & 0 & S+s^{2} T
\end{array}\right)\left(\begin{array}{c}
i_{1} \\
i_{2} \\
x_{1} \\
x_{2}
\end{array}\right) .
$$

The reduced-order model that we propose draws upon using field solutions for each unitary problem at specific frequencies as basis functions to represent the evolution of the electromagnetic field as a function of frequency. Thus, we carry out Galerkin projection onto the reduced-basis space $\mathcal{W}^{N}=\operatorname{span}\left\{\zeta_{1} \equiv\left(\begin{array}{l}x_{1}\left(s_{1}\right) \\ x_{2}\left(s_{1}\right)\end{array}\right), \ldots, \zeta_{N} \equiv\left(\begin{array}{l}x_{1}\left(s_{N}\right) \\ x_{2}\left(s_{N}\right)\end{array}\right)\right\}$, where $s_{1}, \ldots, s_{N}$ are complex frequencies in the frequency band of interest, to obtain the reduced-order linear dynamical system:

$$
\left(\begin{array}{c}
v_{1} \\
v_{2} \\
0 \\
0
\end{array}\right)=\left(\begin{array}{cccc}
0 & 0 & j \tilde{w}_{11} & j \tilde{w}_{12} \\
0 & 0 & j \tilde{w}_{21} & j \tilde{w}_{22} \\
s j \tilde{b} & 0 & \tilde{S}+s^{2} \tilde{T} & 0 \\
0 & s j \tilde{b} & 0 & \tilde{S}+s^{2} \tilde{T}
\end{array}\right)\left(\begin{array}{c}
i_{1} \\
i_{2} \\
\tilde{x}_{1} \\
\tilde{x}_{2}
\end{array}\right) .
$$

In here, the solution vector $\left(\begin{array}{l}x_{1} \\ x_{2}\end{array}\right)$ is approximated by a linear combination of the elements in the reduced-basis space $\mathcal{W}^{N}$, thus, $\left(\begin{array}{l}x_{1} \\ x_{2}\end{array}\right) \approx P \tilde{x}$, where $P=\operatorname{cols}\left\{\zeta_{1}, \ldots, \zeta_{N}\right\}$. As a result, $\tilde{S}=P^{T}\left(\begin{array}{cc}S & 0 \\ 0 & S\end{array}\right) P, \tilde{T}=P^{T}\left(\begin{array}{cc}T & 0 \\ 0 & T\end{array}\right) P$, $\tilde{b}=P^{T}\left(\begin{array}{l}b_{1} \\ b_{2}\end{array}\right)$, and

$$
\begin{aligned}
& \tilde{w}_{11}=b_{1}^{T}\left(x_{1}\left(s_{1}\right) \quad \ldots \quad x_{1}\left(s_{N}\right)\right) \\
& \tilde{w}_{12}=b_{1}^{T}\left(x_{2}\left(s_{1}\right) \quad \ldots \quad x_{2}\left(s_{N}\right)\right) \\
& \tilde{w}_{21}=b_{2}^{T}\left(x_{1}\left(s_{1}\right) \quad \ldots \quad x_{1}\left(s_{N}\right)\right) \\
& \tilde{w}_{22}=b_{2}^{T}\left(x_{2}\left(s_{1}\right) \quad \ldots \quad x_{2}\left(s_{N}\right)\right) .
\end{aligned}
$$

We refer to [4] for a detailed explanation on the actual automatic adaptive selection of the frequency samples $s_{1}, \ldots, s_{N}$ in the frequency band of interest while certifying the accuracy of the reduced order model. As a result, a fully automatic and reliable model order reduction is carried out.

\section{B. Connection Problem}

Let us assume now we have decomposed the analysis domain into two building blocks, namely, block $\mathrm{A}$ and block $\mathrm{B}$, and let us consider the connection problem in order to resemble the whole circuit response. We need to impose tangential continuity of electromagnetic fields at the ports in order to solve for the original circuit response.

Equation (4) is obtained within each bluiding block. Thus, block $\mathrm{A}$ reads:

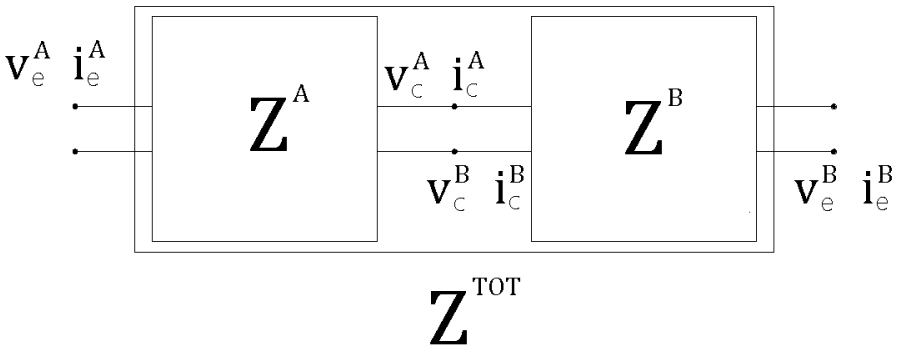

Fig. 1. Building block connection scheme.
$\left(\begin{array}{c}v_{e}^{A} \\ v_{c}^{A} \\ 0 \\ 0\end{array}\right)=\left(\begin{array}{cccc}0 & 0 & j \tilde{w}_{e e}^{A} & j \tilde{w}_{e c}^{A} \\ 0 & 0 & j \tilde{w}_{c e}^{A} & j \tilde{w}_{c c}^{A} \\ s j \tilde{b}^{A} & 0 & \tilde{S}^{A}+s^{2} \tilde{T}^{A} & 0 \\ 0 & s j \tilde{b}^{A} & 0 & \tilde{S}^{A}+s^{2} \tilde{T}^{A}\end{array}\right)\left(\begin{array}{c}i_{e}^{A} \\ i_{c}^{A} \\ \tilde{x}_{e}^{A} \\ \tilde{x}_{c}^{A}\end{array}\right)$,

and block B reads:

$\left(\begin{array}{c}v_{e}^{B} \\ v_{c}^{B} \\ 0 \\ 0\end{array}\right)=\left(\begin{array}{cccc}0 & 0 & j \tilde{w}_{e e}^{B} & j \tilde{w}_{e c}^{B} \\ 0 & 0 & j \tilde{w}_{c e}^{B} & j \tilde{w}_{c c}^{B} \\ s j \tilde{b}^{B} & 0 & \tilde{S}^{B}+s^{2} \tilde{T}^{B} & 0 \\ 0 & s j \tilde{b}^{B} & 0 & \tilde{S}^{B}+s^{2} \tilde{T}^{B}\end{array}\right)\left(\begin{array}{c}i_{e}^{B} \\ i_{c}^{B} \\ \tilde{x}_{e}^{B} \\ \tilde{x}_{c}^{B}\end{array}\right)$.

Finally tangential field continuity at the connected ports implies:

$$
\begin{aligned}
& v_{c}^{A}=v_{c}^{B}=v_{c} \\
& i_{c}^{A}=-i_{c}^{B}=i_{c}
\end{aligned}
$$

which is depicted in Fig. 1. Putting all these equations (6)(8) together results in equation (9), which is the final linear dynamical system for the whole circuit. Following a similar approach as the one described in [6], the final linear dynamical system (9) can be solved in a straightforward way as frequency changes resulting in the GIM of the whole circuit by eliminating the internal state-space variables in the dynamical system.

\section{NUMERICAL RESULTS}

In this section, we apply the proposed technique to different structures, namely, a dual-band circular waveguide filter proposed in [8], and a rectangular waveguide filter used in a waveguide diplexer described in [9]. All computations were carried out on a workstation with $3.00-\mathrm{GHz}$ Intel Xeon ES$2687 \mathrm{~W}$ v 4 processor and 64-GB RAM. FEM simulations were carried out with the code described in reference [9].

First, we use the proposed approach in the analysis of the dual-band circular waveguide filter shown in Fig. 2 and designed in [8], where the geometry details can be found. The analysis domain is decomposed into two subdomains, namely, building block $\mathrm{A}$ and $\mathrm{B}$, which are highlighted in Fig. 2 in different colors (blue and red). The fast frequency sweep connection problem is compared to the results obtained by direct connect and invert results computed frequency by frequency. The results are detailed in Fig. 3. Good agreement is found. It should be noted that the spikes in Fig. 3 are also present in the FEM analysis of this filter. 
Next, we solve for one of the filter channels in a H-plane rectangular waveguide filter designed in [9]. Loss of equiripple return losses is expected after de-embedding this filter channel from the original equi-ripple return losses diplexer results. Once again, the analysis domain is decomposed into two building blocks, namely, block $\mathrm{A}$ and $\mathrm{B}$ highlighted in Fig. 4 in blue and red, respectively. The fast frequency sweep connection problem is compared to the results obtained by direct connect and invert results computed frequency by frequency. These results are detailed in Fig. 5. Good agreement is achieved.

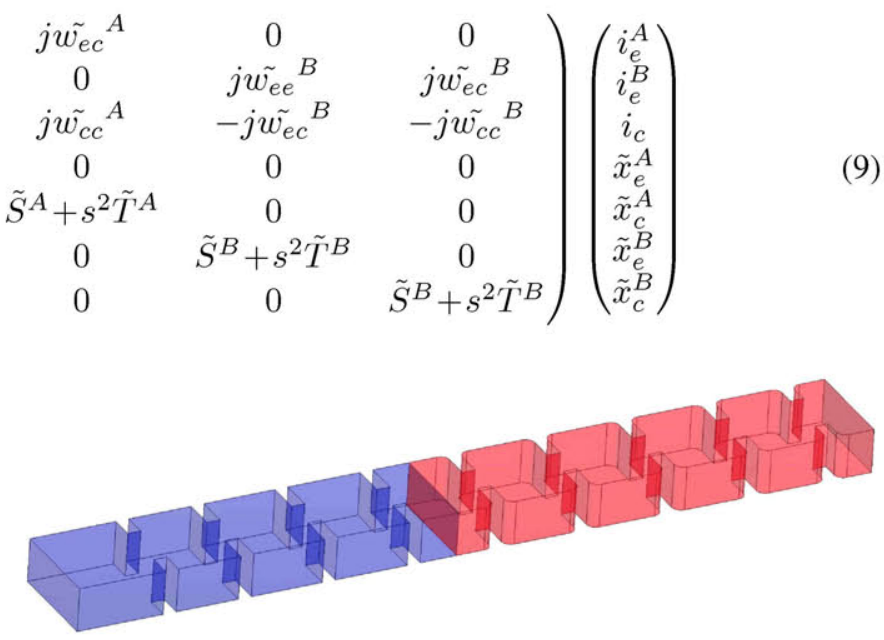

Fig. 4. H-plane rectangular waveguide filter geometry. Different building blocks are taken into account and are highlighted in blue and red.

Fig. 2. Geometry of dual-band circular waveguide filter designed in [8]. Different building blocks are taken into account and are highlighted in blue and red.

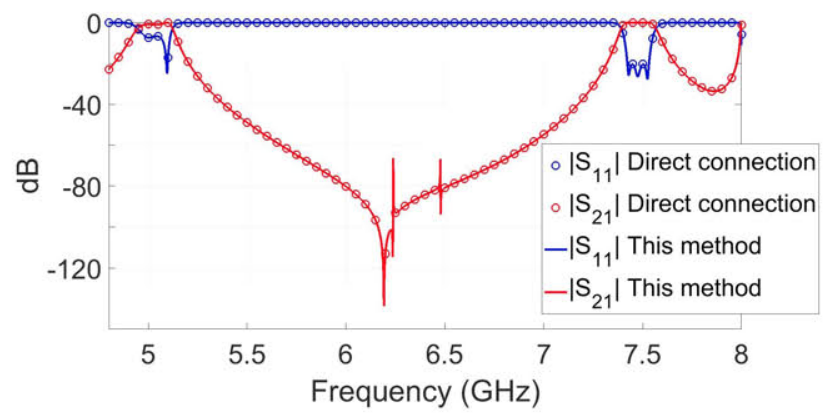

Fig. 3. Dual-band circular waveguide filter fast frequency connection results are compared to direct connection results carried out frequency by frequency.

\section{CONCLUSIONS}

A methodology for fast frequency sweep in connection problems has been described within the framework of modelorder reduction and domain decomposition. Further possibilities for microwave filter analysis can be explored and, in particular, in the design of microwave diplexers.

\section{REFERENCES}

[1] J. Rubio, J. Arroyo, and J. Zapata, "SFELP-an efficient methodology for microwave circuit analysis," IEEE Trans. Microwave Theory Tech., vol. 49, no. 3, pp. 509-516, Mar. 2001.

[2] V. de la Rubia and J. Zapata, "Microwave circuit design by means of direct decomposition in the finite-element method," IEEE Trans. Microwave Theory Tech., vol. 55, no. 7, pp. 1520-1530, Jul. 2007.

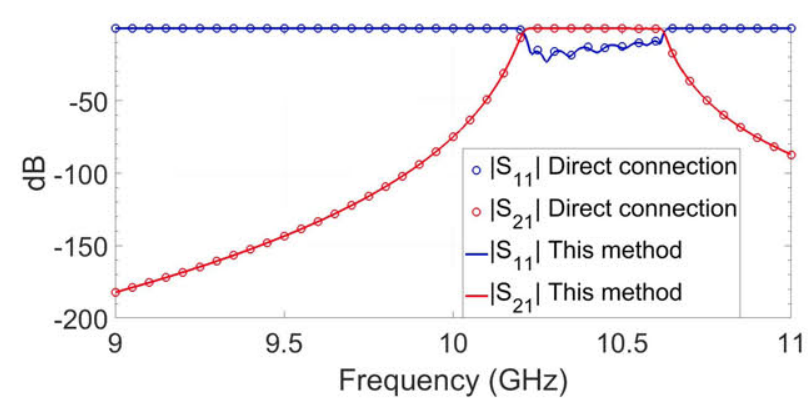

Fig. 5. Rectangular waveguide filter fast frequency connection results are compared to direct connection results carried out frequency by frequency.

[3] A. C. Antoulas, Approximation of Large-Scale Dynamical Systems. Philadelphia, PA: SIAM, 2005.

[4] V. de la Rubia, U. Razafison, and Y. Maday, "Reliable fast frequency sweep for microwave devices via the reduced-basis method," IEEE Trans. Microwave Theory Tech., vol. 57, no. 12, pp. 2923-2937, Dec. 2009.

[5] M. Rewienski, A. Lamecki, and M. Mrozowski, "A goal-oriented error estimator for reduced basis method modeling of microwave devices," IEEE Microwave Wireless Compon. Lett., vol. 25, no. 4, pp. 208-210, Apr. 2015.

[6] P. Arcioni and G. Conciauro, "Combination of generalized admittance matrices in the form of pole expansions," IEEE Trans. Microwave Theory Tech., vol. 47, no. 10, pp. 1990-1996, Oct. 1999.

[7] G. Conciauro, M. Guglielmi, and R. Sorrentino, Advanced Modal Analysis. New York, NY: Wiley, 2000.

[8] U. Naeem, S. Bila, M. Thvenot, T. Mondire, and S. Verdeyme, "A dual-band bandpass filter with widely separated passbands," IEEE Trans. Microwave Theory Tech., vol. 62, no. 3, pp. 450-456, Mar. 2014.

[9] A. Lamecki, L. Balewski, and M. Mrozowski, "An efficient framework for fast computer aided design of microwave circuits based on the higherorder 3d finite-element method," Radioengineering, vol. 23, no. 4, 2014. 